Citation: F.S. Rota, M. Bagliani, P. Feletig (2020). Pattern regionali e demografici del Covid-19 durante la prima ondata pandemica in Italia. Proposta di uno studio pilota per l'applicazione della metodologia shift-share alla dinamica delle infezioni. Bollettino della Società Geografica Italiana serie 14, 3(2): 25-38. doi: 10.36253/bsgi-1153

Copyright: (c) 2020 F.S. Rota, M. Bagliani, P. Feletig. This is an open access, peer-reviewed article published by Firenze University Press (http://www. fupress.com/bsgi) and distributed under the terms of the Creative Commons Attribution License, which permits unrestricted use, distribution, and reproduction in any medium, provided the original author and source are credited.

Data Availability Statement: All relevant data are within the paper and its Supporting Information files.

Competing Interests: The Author(s) declare(s) no conflict of interest.

For Italian evaluation purposes: Francesca Silvia Rota takes responsability for sections 2 and 4, Marco Bagliani for sections 1 and 3, and Paolo Feletig for section 5 and shift-share calculations.

\section{Pattern regionali e demografici del Covid-19 durante la prima ondata pandemica in Italia. Proposta di uno studio pilota per l'applicazione della metodologia shift-share alla dinamica delle infezioni}

\author{
Covid-19 regional and demographic patterns during the first \\ wave in Italy. A proposal for a pilot study applying the shift-share \\ analysis to the dynamic of the infections
}

\author{
Francesca Silvia Rota ${ }^{1}$, Marco Bagliani ${ }^{2}$, Paolo Feletig ${ }^{3}$ \\ ${ }^{1}$ IRCrES CNR - Istituto di Ricerca sulla Crescita Economica Sostenibile, Moncalieri, Italia \\ ${ }^{2}$ Università degli Studi di Torino, Dipartimento di Economia e Statistica "Cognetti de \\ Martiis". Centro interuniversitario IRIS (Istituto di Ricerche Interdisciplinari sulla Soste- \\ nibilità). Torino, Italia \\ ${ }^{3}$ IRES Piemonte - Istituto Ricerche Economico Sociali del Piemonte, Torino, Italia \\ E-mail: francesca.rota@ircres.cnr.it,marco.bagliani@unito.it, feletig@ires.piemonte.it
}

\begin{abstract}
The paper presents the experimental attempt to apply the shift-share decomposition technique, mainly used in the economic field to analyse regional differentials, to the growth dynamics of infections during the first wave of the COVID-19 pandemic. Through a partial readjustment of the initial formulations of this technique, the regional patterns of the spread of the infections in Italy are analysed, taking into account the influence exercised by the demographic characteristics (age composition) of the region. In this reformulation, the shift-share analysis (SSA) allows to break down the daily variation of COVID-19 cases according to four effects resulting from: the distribution of the population by age groups (measured through the demographic and allocative effects), the tendency of the regional dynamics to follow the trend of the nation (measured by the national effect) and the rising of specific local dynamics (measured by the local effect). The application of our proposed reformulation studies the diffusion of infections in the Italian regions between March 9 and May 20, 2020, highlighting strengths and weaknesses of the methodology, offering ideas for further development and refinements to use SSA for applications in extra-economic realms (demographic, epidemiologic etc.), fruitfully. For example, the choice of the Italian case study was detrimental to the quality of the results obtained, since in Italy the population's age distribution tends to be similar. For this reason, at the end of our study, it is suggested the opportunity to test the robustness of the proposed method using as case study other European nations (for example, France, Spain or Germany) characterised by more significant heterogeneity of the regional population than Italy.
\end{abstract}

Keywords: COVID-19, age structure, registered infections, shift-share, Italian regions. 
Riassunto. Il lavoro presenta la proposta, ancora in una fase sperimentale, di applicare la tecnica di scomposizione shift-share, prevalentemente utilizzata in ambito economico per l'analisi dei differenziali di crescita, alla dinamica delle infezioni durante le fasi iniziali della pandemia di COVID-19. Tramite un originale aggiustamento metodologico della formulazione dinamico-cumulativa, il presente articolo analizza i pattern regionali di diffusione dell'infezione COVID-19 in Italia, tenendo conto dell'influenza che su di essi esercitano le caratteristiche demografiche (distribuzione per classi di età) della popolazione residente. Nella riformulazione qui proposta, la shift-share analysis (SSA) permette di scomporre la variazione giornaliera dei casi di COVID-19 in funzione: della distribuzione della popolazione per classi di età (misurata attraverso gli effetti demografico e allocativo); della maggiore o minore tendenza della dinamica regionale a seguire il trend della nazione (misurata dall'effetto nazionale) e dell'insieme delle caratteristiche endogene della regione che possono favorire o limitare l'incidenza del virus sul territorio rispetto al resto della nazione (misurata dall'effetto locale). Dallo studio condotto sull'andamento dei contagi nelle regioni italiane tra il 9 marzo 2020 e il 20 maggio 2020 emergono punti di forza e limiti della metodologia qui proposta, che offrono spunti per un ulteriore sviluppo delle applicazioni in ambito extra economico (demografico, epidemiologico ecc.) della SSA. Per esempio, pregiudizievole per la qualità dei risultati ottenuti è stata la scelta del caso studio dell'Italia, perché è caratterizzata da una distribuzione per età della popolazione tendenzialmente omogenea nelle diverse regioni. Per questo, a conclusione dello studio, si suggerisce l'opportunità di testare la robustezza del metodo proposto con riferimento ad altre nazioni europee (per esempio la Francia, la Spagna o la Germania) caratterizzate da una maggiore eterogeneità anagrafica della popolazione regionale rispetto all'Italia.

Parole chiave: COVID-19, struttura dell'età, infezioni registrate, shift-share, regioni italiane.

\section{Introduzione.}

Il diffondersi della prima ondata pandemica dell'infezione da COVID-19 ha portato ad una forte produzione scientifica orientata a studiare l'origine, l'evoluzione e le ricadute di questa specifica malattia e della drammatica crisi ad essa associata. In questo processo, le scienze geografiche e regionali hanno fornito il proprio contributo, focalizzando l'attenzione soprattutto sullo studio degli aspetti socio-culturali e territoriali della pandemia. Il presente articolo assume come riferimento il filone più quantitativo di questa letteratura, proponendo un'applicazione inedita della metodologia di scomposizione shift-share. Di norma utilizzata nello studio dei differenziali di crescita economica tra i territori, questa tecnica viene qui proposta per esaminare, in termini compara- ti, la dinamica giornaliera delle infezioni da COVID-19, registrate nelle regioni italiane durante la prima ondata. Grazie alla shift-share diventa possibile, da un lato, quantificare il ruolo che la struttura demografica ha nel favorire o limitare l'incidenza del virus sul territorio e, dall'altro lato, stimare, seppur in termini aggregati, se e quanto l'insieme dei fattori endogeni alle regioni (per esempio politiche, densità della popolazione, comportamenti individuali e collettivi) influiscono sulla diffusione dei contagi. Obiettivo del presente lavoro è quindi verificare se, in Italia, durante la prima ondata pandemica, esiste una influenza della componente demografica sulla diffusione del virus alla scala regionale e separarla da quella di altri fattori endogeni alla regione. Al meglio della nostra conoscenza, è questo un tentativo che non trova precedenti nella letteratura geografica e regionale.

Ci si muove in un terreno di studio nuovo, a cavallo fra la scienza regionale e scienza medica, reso accidentato dalla complessità del fenomeno analizzato. Rispetto alla statistica medica che, non di rado, nello studiare i fattori patogeni della malattia introduce anche considerazioni di tipo territoriale (Wolkewitz, Puljak 2020), l'analisi qui proposta se ne discosta sia da un punto di vista formale (come si dirà, l'equazione matematica della shift-share da cui si è partiti è una scomposizione che non prevede il ricorso a procedure di regressione e di analisi multivariata), quanto da un punto di vista interpretativo, giacché il fine dello studio è discriminare le modalità con cui la diffusione del virus si è concretizzata nelle regioni italiane rispetto alla dinamica media nazionale.

Nel caso del COVID-19, i tassi di letalità (proporzione percentuale tra il numero dei decessi sul totale dei soggetti ammalati) e mortalità (proporzione tra i decessi e la popolazione suscettibile) sono, da subito, risultati significativamente correlati con l'età. Mentre per quel che riguarda l'influenza del dato anagrafico sulla morbilità (proporzione tra i malati e la popolazione totale), ad oggi non ci risulta siano state ancora condotte delle analisi esaustive sulla probabilità con cui il virus si diffonde in individui di età diversa. Soprattutto, non ci risulta esistano studi che considerano contemporaneamente la variabile anagrafica e quella territoriale. La trattazione qui proposta, riferita alla diffusione delle infezioni da COVID-19 tra le regioni italiane, vuole essere un primo passo in questa direzione.

Il paragrafo 2 focalizza l'attenzione sugli studi che, all'interno della recente letteratura sul COVID-19, identificano nella variabile anagrafica un fattore di spiegazione della diffusione delle infezioni. Il paragrafo 3 illustra la riformulazione della metodologia shift-share adottata per l'analisi, mentre il paragrafo 4 discute i risultati ottenuti. Chiude il contributo il paragrafo 5 con una discus- 
sione sulla validità e sui limiti della sperimentazione attuata, sulle ricadute dei risultati ottenuti e sulle prospettive future di ricerca.

\section{La variabile anagrafica nella spiegazione della diffusione del COVID-19}

L'eccezionalità della malattia e della sua diffusione, insieme con la gravità delle ripercussioni ad essa connesse, hanno fatto del COVID-19 un prioritario ambito di ricerca per gli studiosi di tutte le discipline. Rispetto al trend generale, la letteratura regionalista e territorialista pone al centro dell'attenzione soprattutto due questioni (Pisano et al. 2020):

- l'identificazione dei fattori territoriali utili a spiegare le marcate differenze geografiche di diffusione delle infezioni e dei decessi da SARS-CoV-2;

- la misurazione degli impatti e dei divari causati dalla diffusione del virus e dalle misure per il suo contenimento.

In entrambi i casi le variabili prese in considerazione sono molte e diversificate. Per esempio, con riferimento ai determinanti della diffusione del COVID-19, si passa dallo studio dell'occupazione e dal valore aggiunto regionale, alla mobilità pendolare e studentesca, dalla prossimità spaziale e sociale tra gli individui, alla presenza di strutture di cura e posti letto e finanche all'inquinamento atmosferico (Musolino, Rizzi 2020; Kubota et al. 2020). Né è mancata l'attenzione per la diversa efficacia dimostrata dalle politiche pubbliche nazionali e regionali nel contrastare la diffusione delle infezioni (Cohen, Kupferschmidt 2020).

Il presente contributo si inserisce nel filone di studi sulle determinanti della diffusione dei casi di COVID-19, concentrando l'attenzione sull'influenza delle variabili demografiche e in particolare della distribuzione della popolazione per classi di età. Nello stesso tempo, esso se ne differenzia per lo specifico taglio metodologico innovativo assunto nella trattazione.

L'interesse per il dato anagrafico si spiega in ragione della diffusa percezione che, sin dalle prime fasi della pandemia, identificava negli anziani la categoria più esposta e bisognosa di tutele (Kluge 2020). Ma se con riferimento al rischio di decesso, i dati forniti da ISS e WHO da subito evidenziano l'elasticità esistente tra l'età delle persone infettate e i tassi di mortalità e letalità del COVID-19 (Kluge 2020; ISS 2020b), sull'incidenza dell'età avanzata rispetto al rischio di contrarre l'infezione i risultati della ricerca non sono univoci (Dudel et al. 2020) e il dibattito resta aperto (Dowd et al. 2020).
A parte alcune eccezioni (Kubota et al. 2020), nell'analizzare la diffusione del COVID-19, l'età emerge in genere come concausa secondaria, non primaria (Nepomuceno et al. 2020). Oltre che a condizioni di comorbilità, obesità e patologie croniche, che rendono critico il decorso della malattia (ISS 2020b), l'età avanzata risulta per esempio associata a una maggiore frequenza di situazioni rischiose di esposizione, quali il risiedere ospedali, case di cura e residenze per anziani (ISTAT 2020; Lombardo 2020), il muoversi prevalentemente con i mezzi pubblici (ISTAT 2018) o, ancora, il prendersi cura quotidianamente dei figli e dei nipoti (Sarti 2010).

Per l'analisi della diffusione della pandemia, considerare la distribuzione per classi di età della popolazione nei vari contesti regionali potrebbe rivelarsi un significativo ambito di studio (Bignami-Van Assche et al. 2020). Ciò nondimeno, la letteratura risulta un po' carente da questo punto di vista. Gli studi rivolti a verificare il peso della struttura demografica sulla diffusione della prima ondata del COVID-19 sono pochi e in genere focalizzati sul ruolo "trainante" delle infezioni tra la popolazione anziana, mentre altri studi sostengono che le fasce di età che più hanno guidato la dinamica di crescita della curva cumulativa delle infezioni nella primavera del 2020 siano state quelle dei 50-59enni e dei 60-69enni (Bignami-Van Assche et al. 2020). Il tema è evidentemente complesso e, in assenza di studi che dimostrino l'esistenza di una chiara correlazione tra l'età e la morbilità del COVID-19 (Dowd et al. 2020), non stupisce che anche nel calcolare gli indici utilizzati dall'OMS per monitorare la velocità di trasmissione del virus, $R_{0}$ (calcolato nella fase iniziale dell'epidemia) e $R_{t}$ (calcolato successivamente all'entrata in vigore delle misure di contenimento), l'età non sia presa considerazione, ma venga "relegata" dalla statistica medica e epidemiologia al ruolo di confounding factor, che "confonde" l'associazione fra esposizione e esito della dinamica osservata (Daniel, Cross 2019).

\section{Proposta per una applicazione in ambito non economico della metodologia shift-share}

L'analisi shift-share (SSA) è da diversi decenni «il metodo più comunemente utilizzato per misurare le componenti nazionali, industriali e regionale degli scostamenti registrati nei tassi regionali di variazione dell'occupazione [e della ricchezza; ndr]» (Patterson 1991, 211, traduzione degli autori). Proposta nel 1959 da Dunn, e in seguito sviluppata in numerose varianti (documentate, tra gli altri, da: Sihag, McDonough 1989; Fernàndez, Menàndez 2005; Artige, van Neuss 2014; Lahr, Ferreira 2020), questa tecnica è solitamente impie- 
gata quando si è in presenza di un sistema territoriale (tipicamente di scala nazionale) suddiviso in sottoambiti (ad esempio le regioni) e si vuole scomporre la variazione temporale di una grandezza socio-economica di interesse (ad esempio gli occupati o il valore aggiunto) in singoli effetti tra loro complementari. Il fine è cogliere informazioni:

- sulla performance regionale rispetto a quella nazionale;

- sugli effetti delle differenze tra la composizione settoriale regionale e quella nazionale;

- sulla specifica capacità di crescita della regione rispetto alla nazione.

La SSA ha ormai alle spalle una tradizione corposa di modificazioni successive, che hanno permesso di affinarne il funzionamento e risolvere molti dei limiti iniziali. Tra gli sviluppi principali, in particolare, si ricordano i contributi di Barff e Knight (1988) e di Esteban-Marquillas (1972), volti a migliorare la solidità dell'equazione di scomposizione e le recenti rivisitazioni in chiave dinamico-cumulativa (Artige, van Neuss 2014). A questi si aggiungono le riformulazioni di tipo probabilistico (Patterson 1991) e quelle a struttura spaziale, capaci di discriminare in funzione delle interazioni tra le unità territoriali analizzate. A partire dalla matrice di modellizzazione delle relazioni territoriali proposta da Nazara e Hewings (2004), sono infatti numerose le soluzioni offerte da questo specifico tipo di SSA per la pesatura spaziale delle variabili (cfr. tra gli altri: Fernàndez, Menàndez 2005).

I processi analizzati con la metodologia shift-share continuano però ad essere soprattutto quelli economici, mentre, a conoscenza degli autori, sono rari gli studi che considerano fenomeni "extraeconomici" (demografici, epidemiologici ecc.). In altre parole, oggi si perde l'occasione di estendere a altri ambiti significativi di analisi le interessanti prospettive di lettura ed interpretazione temporale, spaziale e strutturale dei dati che la scomposizione shift-share dimostra di possedere.

Nel proporre quindi l'applicazione della shiftshare all'analisi della dinamica nel tempo dei casi di COVID-19, si è deciso di utilizzare una metodologia di SSA che non prendesse in considerazione la pesatura spaziale. La natura sperimentale dell'esercizio proposto e la complessità delle variabili in gioco (epidemiologiche, sociali, territoriali, spaziali) hanno infatti consigliato di partire dalla scomposizione secondo il modello "classico", in favore della quale muove anche il fatto che, per ampia parte del periodo considerato, la mobilità e l'interazione inter-regionale in Italia sia quasi del tutto annullata dal primo lockdown nazionale.

Come si è detto, uno dei vantaggi principali dell'applicazione della SSA alla diffusione del COVID-19 può essere identificato nella capacità di separare i contributi strutturali da quelli territoriali che concorrono a spiegare la dinamica analizzata. Un altro pregio è quello di consentire un approccio sistematico e coerente per la misura della differente intensità del fenomeno indagato a scala sub-nazionale rispetto a quella nazionale (Conti Puorger 2018).

Gli svantaggi risiedono invece nella natura deterministica dell'equazione shift-share (Patterson 1991) che si sviluppa a partire da una identità matematica con tre sole variabili (in questo caso: il numero delle infezioni accertate, la regione di riferimento e la classe di età della popolazione). Ciò comporta una forte semplificazione che, in un contesto complesso come quello della diffusione di una malattia epidemica, va a svantaggio della piena comprensione del fenomeno analizzato. Come verrà più sotto ricordato, si vuole comunque sottolineare che lo scopo del presente scritto non è di offrire una spiegazione esaustiva dei diversi driver alla base della diffusione dell'epidemia, quanto piuttosto mettere alla prova una metodologia che consenta, in modo semplice, di separare e stimare quanto contano gli aspetti demografici rispetto ad altri fattori endogeni alla regione.

La limitata qualità delle informazioni disponibili è un altro problema riconosciuto nello sviluppo delle analisi sul COVID-19 (Pisano et al. 2020): nei paesi ricchi come in quelli poveri, i dati ufficiali disponibili sulla diffusione dei COVID-19 spesso presentano problemi di affidabilità, omogeneità, tempestività e livello di dettaglio (Lloyd-Sherlock et al. 2021; Wolkewitz, Puljak 2020).

Per adattare la shift-share all'analisi della diffusione della prima ondata di COVID-19, viene qui proposta una parziale riformulazione della metodologia. In questo caso, il ruolo giocato dalla composizione settoriale dell'economia viene sostituito dalla struttura demografica della popolazione, suddivisa per fasce d'età, mentre la capacità di mantenere basse le curve di diffusione del virus all'interno dei territori regionali prende il posto della competitività (ossia della capacità di incrementare il numero di occupati o il valore aggiunto). La sostituzione della composizione strutturale di tipo economico con quella anagrafica avviene sulla base di una ipotesi di ricerca che, riconoscendo alla variabile dell'età una influenza significativa sui livelli di mortalità e letalità del COVID-19 (ISS 2020a), vuole verificarne la relazione (non ancora dimostrata dalla letteratura) anche rispetto alla morbilità, ossia alla probabilità che gli individui hanno di contrarre la malattia. Anche per quel che riguarda il passaggio dai differenziali regionali di crescita economica alla diversa resistenza alla diffusione dell'epidemia, si tratta di una proposta inedita, che trova un parziale inquadramento teorico nella nuova attenzio- 
ne della geografia per il concetto di resilienza territoriale in abbinamento a quello di competizione (Bristow 2010).

Tra i principali limiti delle analogie proposte vi sono certamente: il diverso peso relativo e il differente dinamismo dei settori economici (molto diversificati da regione a regione) rispetto a quelli che caratterizzano la distribuzione della popolazione per classi demografiche (che sono molto simili tra le regioni); la diversa natura dei raggruppamenti, tale per cui le classi anagrafiche sottendono una medesima "biologia" (che, a parità di condizioni, porta a comportamenti territorialmente simili) e "rapporti di proporzione" che si spiegano in ragione di macroprocessi demografici (invecchiamento, riduzione della natalità, variazione dell'immigrazione).

Questi fatti, insieme con l'osservazione della limitata differenziazione regionale delle piramidi delle età in Italia (di cui si dirà nel commentare i risultati dell'analisi; cfr. Fig. 4), suggeriscono evidentemente di non considerare generalizzabili gli esiti di una sperimentazione che, cogliendo l'opportunità della disponibilità dei dati forniti dall'ISS, è stata sviluppata in prima istanza sul caso italiano, ma che potrebbe essere proficuamente applicata anche in altri Paesi.

La diversa natura della variabile considerata (infezioni al posto di occupati o valore aggiunto) e il diverso significato assunto da una dinamica di crescita di quest'ultima (crisi sanitaria al posto di crescita economica) impongono aggiustamenti delle equazioni matematiche alla base della shift-share. La versione di partenza, utilizzata per la riformulazione della metodologia, è la shift-share dinamico-cumulativa descritta in Bagliani e collaboratori (2019) e Rota e collaboratori (2020), che sviluppa e potenzia la versione precedentemente elaborata dagli studi di Esteban-Marquillas (1972). La scelta della variabile da analizzare è cruciale per una corretta formulazione: per studiare la diffusione del COVID-19 non conviene utilizzare il numero di malati, ma il numero delle persone che rimangono "sane", ossia delle persone per le quali non esiste una infezione documentata al COVID-19. Questa scelta, apparentemente controintuitiva, è giustificata per evitare di avere variazioni percentuali del numero di malati infinite o comunque esageratamente elevate ${ }^{1}$, criticità che non emerge se si considera il numero dei non infetti (comprensivo di coloro che non si sono sottoposti a tampone o che, avendolo fatto, sono risultati negativi).

Si consideri quindi la variabile di riferimento $E_{i r}{ }^{t}$ che rappresenta il numero di persone della regione $r$ e del-

\footnotetext{
${ }^{1}$ Passando da 0 a 1 malato si ha una variazione percentuale del numero di malati infinita, così come passando da 1 a 10 malati tale variazione percentuale è pari al $1000 \%$.
}

la classe d'età $i$ che, al tempo $t$, non risultano positive al COVID-19. L'analisi così ridefinita scompone la variazione, intercorsa nel periodo tra $t_{0}$ e $t_{1}$, della popolazione sana in quattro effetti descritti dalla seguente equazione:

$\Delta E_{i r}^{t 0}=E_{i r}^{t 1}-E_{i r}^{t 0}=N E_{i r}{ }^{t 0}+D E_{i r}{ }^{t 0}+L E_{i r}{ }^{t 0}+A E_{i r}{ }^{t 0}$

I quattro effetti sono definiti come segue:

$$
\begin{aligned}
& N E_{i r}{ }^{t 0}=E_{i r}^{* t 0} g_{i N}{ }^{t 0} \\
& D E_{i r}{ }^{t 0}=\left(E_{i r}{ }^{t 0}-E_{i r}^{*}{ }^{t 0}\right) g_{i N}{ }^{t 0} \\
& L E_{i r}{ }^{t 0}=E_{i r}^{*}{ }^{t 0}\left(g_{i r}{ }^{t 0}-g_{i N}{ }^{t 0}\right) \\
& A E_{i r}{ }^{t 0}=\left(E_{i r}{ }^{t 0}-E_{i r}^{*}\right)\left(g_{i r}{ }^{t 0}-g_{i N}{ }^{t 0}\right)
\end{aligned}
$$

dove il pedice $N$ indica il valore aggregato nazionale e: $E_{r}{ }^{t 0}=\sum_{i} E_{i r}{ }^{t 0}$ e $E_{N}{ }^{t 0}=\sum_{i} E_{i N}{ }^{t 0}$, rispettivamente indicano il numero totale di non infetti nella regione $r$ e nella nazione al tempo $t_{0}$.

$E_{i r}^{*}{ }^{t 0}=\left(E_{r}^{t 0}\right)\left(E_{i N}{ }^{t 0} / E_{N}{ }^{t 0}\right)$ indica il numero omotetico di persone non infette che la regione $r$ avrebbe, nella fascia d'età $i$, se questa avesse la stessa composizione demografica media nazionale.

$g_{i N}{ }^{t 0}=\left(E_{i N}{ }^{t 1}-E_{i N}{ }^{t 0}\right) / E_{i N}{ }^{t 0}$ indica il tasso di variazione dei non infetti nella fascia d'età $i$ al livello nazionale nel periodo compreso tra $t_{0}$ e $t_{1}$. Nelle nostre analisi questo valore risulta sempre negativo poiché rappresenta la diminuzione del numero dei non infetti, coerentemente con la fase di diffusione della malattia esaminata nel presente articolo.

$g_{i r}{ }^{t 0}=\left(E_{i r}{ }^{t 1}-E_{i r}{ }^{t 0}\right) / E_{i r}{ }^{t 0}$ descrive il tasso di variazione dei non infetti nella fascia d'età $i$ nella regione $r$ nel periodo compreso tra $t_{0}$ e $t_{1}$. In tutte le regioni italiane questo tasso assume valori negativi che possono a loro volta risultare inferiori o superiori al valore nazionale $g_{i N}{ }^{t 0}$, coerentemente con la diversa diffusione della malattia che caratterizza le regioni rispetto alla media italiana.

Al fine di rendere le comparazioni tra regioni più agevoli ogni effetto calcolato dalla metodologia è espresso in termini percentuali rispetto al tempo base $t_{0}$ dividendo ogni fattore della formula (1) per $E_{i r}{ }^{t 0} .^{2}$

I quattro effetti della scomposizione hanno i significati qui di seguito descritti.

- $\quad N E_{i r}{ }^{t 0}$ (da ora in poi NE). L'effetto nazionale misura la variazione del numero dei non infetti che si avrebbe nel caso in cui la struttura demografica regionale e le caratteristiche regionali che favoriscono o inibiscono la diffusione del virus fossero uguali a quelle medie nazionali. Questo effetto, come ben

\footnotetext{
${ }^{2}$ Per ulteriori approfondimenti sulla metodologia dinamico-cumulativa
} si rimanda a Rota et al. (2020). 
osservabile dalla formula (2), risulta sempre negativo poiché proietta sulle regioni il tasso di diffusione medio della malattia (ossia di riduzione del numero dei sani) a livello nazionale.

- $D E_{i r}{ }^{t 0}$ (da ora in poi DE). L'effetto demografico indica l'influenza della struttura demografica regionale sulla diffusione del virus, ovvero quantifica la differenza nell'evoluzione del numero dei non infetti causata dalle diverse distribuzioni della popolazione in classi di età tra regioni e nazione.

- $L E_{i r}{ }^{t 0}$ (da ora in poi LE). L'effetto locale considera le caratteristiche endogene della regione che, a parità di struttura demografica, favoriscono o inibiscono la diffusione dell'epidemia rispetto a quanto accade mediamente nel resto della nazione. Si riferisce quindi in modo aggregato a diverse tipologie di fattori specificamente regionali, come ad esempio: politiche di contenimento, densità abitativa, presenza di particolari condizioni dell'ambiente fisico, differenti abitudini culturali, ecc.

- $A E_{i r}{ }^{t 0}$ (da ora in poi AE). L'effetto allocativo quantifica quella che potrebbe essere definita come l'efficienza (o inefficienza) della struttura demografica regionale nel contenere la diffusione del virus. Attraverso di esso è possibile capire come la distribuzione demografica della popolazione regionale si ripartisca tra le classi di età più o meno colpite dai contagi a livello regionale. Questo effetto è negativo (indicando una inefficienza regionale nel contenere il virus) quando la regione presenta una struttura demografica maggiormente distribuita fra le fasce di popolazione più esposte ai contagi oppure nel caso di una minore distribuzione della popolazione tra le classi di età per le quali si registra una percentuale di contagi minore. L'effetto risulta invece positivo (indicando una efficienza regionale nel limitare la diffusione del virus) nel caso si abbiano combinazioni opposte dei fattori.

Il caso studio qui preso in considerazione riguarda le infezioni da SARS-CoV-2 registrate in Italia tra il 9 marzo e il 20 maggio 2020, stratificate per regioni e per classi di età. Fonte dei dati utilizzati nelle elaborazioni sono state le Appendici con dettaglio regionale allegate ai Report "Epidemia COVID-19" sulla situazione in Italia, rilasciate con cadenza inizialmente bisettimanale, quindi settimanale, dall'Istituto Superiore di Sanità (ISS). In particolare, si sono considerati tutti i report pubblicati fino al 20 maggio 2020 (ISS 2020a), perché, dalle edizioni successive, i dati sulle infezioni hanno smesso di essere presentati per classi di età. I dati pubblicati sono stati riportati a una serie temporale giornaliera attraverso una procedura di interpolazione matematica di tipo lineare.

\section{Risultati dell'analisi}

Nella trattazione che segue la diffusione della malattia è quantificata a partire dal numero delle persone per le quali non è stata certificata la presenza della malattia, ossia il totale della popolazione presente al 31 dicembre 2019 al netto del numero delle persone risultate positive al COVID-19.

Dall'analisi delle componenti shift-share, di cui i grafici che seguono mostrano la dinamica nel tempo (Figg. 1, 2 e 3), emerge un risultato netto e coerente: una quasi totale assenza di significative differenze territoriali imputabili al dato anagrafico. Ciò si evince dall'andamento delle componenti DE e AE: quasi sempre residuale rispetto a quello giocato dalle componenti NE e LE. Il forte gradiente Nord-Sud che ha connotato la diffusione dei contagi nel Paese durante la prima ondata (Musolino, Rizzi 2020; Ascani et al. 2021) viene quindi a dipendere quasi esclusivamente dalle peculiari condizioni (anche non strutturali, come nel caso dei diversi livelli di circolazione del virus presenti all'inizio del lockdown o delle diverse politiche di test e tracciamento intraprese) dei sistemi regionali e non dal peso che le varie coorti di età giocano all'interno delle regioni.

Una prima evidenza che emerge dal confronto di tutti i grafici riguarda infatti il diverso impatto in termini di infezioni raggiunto a maggio. La quota maggiore di casi per abitante si registra nella Provincia Autonoma di Trento, con una diminuzione percentuale delle persone non infette (che riflette un aumento di infetti) pari allo 0,98\% della popolazione complessiva, e in Valle d'Aosta, con una incidenza del 0,94\%. Seguono i casi di Lombardia $(0,79 \%)$ e Piemonte $(0,69 \%)$ che presentando però una popolazione molto maggiore e hanno quindi subito un impatto significativamente maggiore in valore assoluto. All'opposto, nel Sud del Paese, in regioni quali la Basilicata, la Calabria e la Sicilia, così come anche in Campania e in Sardegna, l'incidenza delle persone infettate nel corso della prima ondata dell'epidemia è rimasta su valori molto più bassi (tra lo $0,6 \%$ e lo $0,8 \%$ della popolazione regionale), residuali rispetto a quelli del Nord.

Con riferimento a queste regioni, i grafici mostrano una situazione piuttosto omogenea dal punto di vista del peso delle diverse componenti della dinamica delle infezioni. In tutti i grafici riportati (Figg. 1, 2 e 3), infatti, per spiegare l'andamento della curva risultante basta considerare i soli effetti nazionale (NE) e competitivo (LE), generalmente entrambi di valore negativo.

Fanno eccezione i casi della Liguria e del FriuliVenezia Giulia (Fig. 1) in cui si evidenzia un piccolo contributo dell'effetto demografico DE. La struttura per 

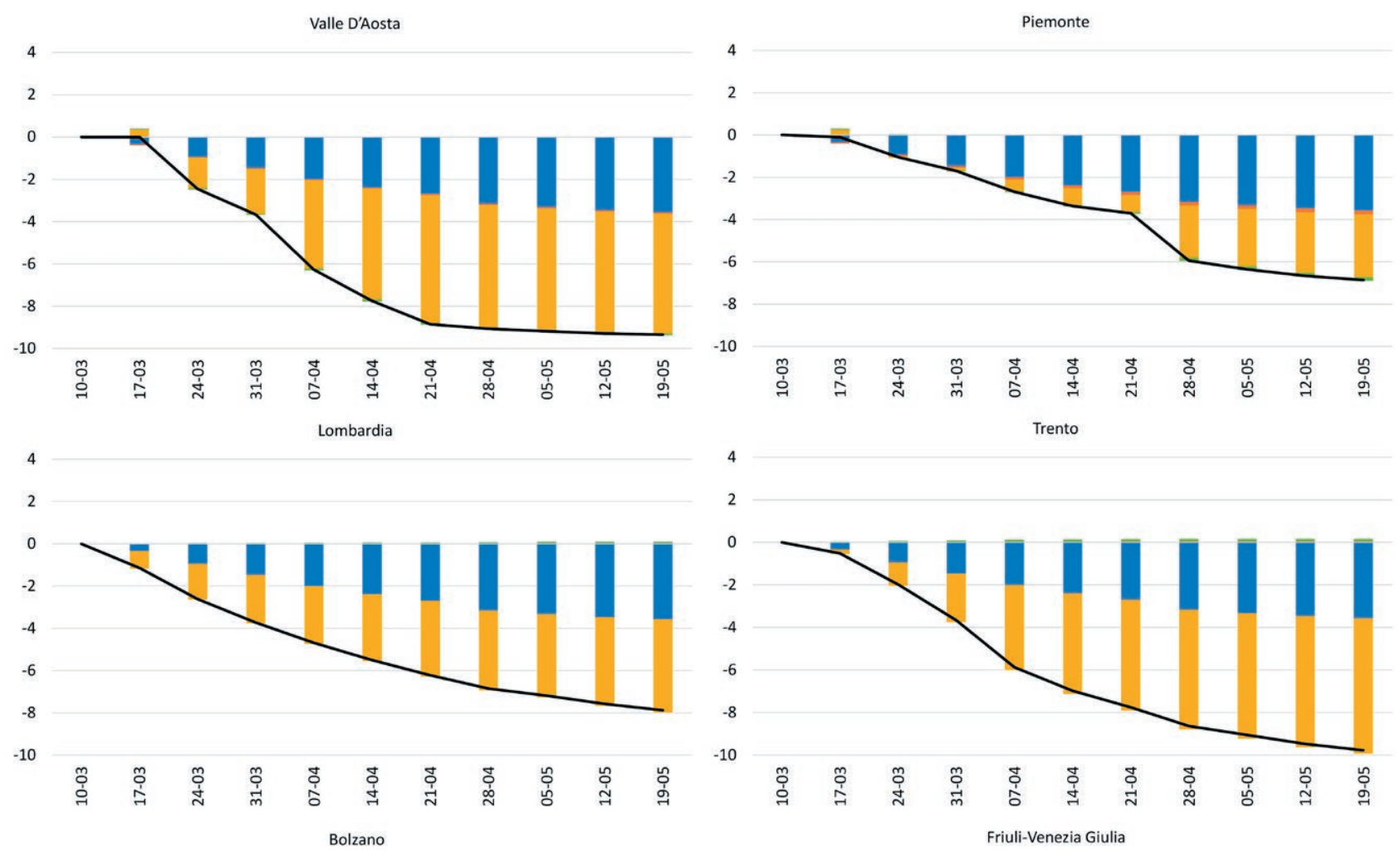

Figura 1a. Shift-share della dinamica delle infezioni da COVID-19 certificate in Italia nelle regioni settentrionali: Valle d'Aosta, Piemonte, Liguria, Lombardia, Provincia Autonoma di Trento, Provincia Autonoma di Bolzano, Veneto, Friuli-Venezia Giulia, Emilia-Romagna. La linea nera rappresenta la diminuzione percentuale del numero di persone non infette. Periodo: 9 marzo - 20 maggio 2020. Fonte: nostre elaborazioni su dati ISS.

fasce d'età di queste regioni, caratterizzata da una forte incidenza di popolazione anziana, potrebbe in particolare spiegarne la maggiore vulnerabilità alla diffusione del virus. Il caso ligure, è inoltre peculiare perché l'effetto competitivo (LE) passa da positivo a negativo, più o meno a metà del periodo analizzato. Un cambio repentino che determina una discontinuità evidente anche nella curva della variazione delle infezioni, e che noi assumiamo sia imputabile non tanto a una modificazione strutturale della compagine regionale, quanto a una modifica sostanziale nell'attività di rilevazione delle infezioni, che solo a fine aprile riesce a documentare in modo più preciso il reale andamento della malattia.

Una modesta componente positiva di LE si rileva anche per il Friuli-Venezia Giulia. In altre parole, a fronte di una situazione similmente svantaggiata dal punto di vista anagrafico, nel solo caso del Friuli-Venezia Giulia una qualche forma di vantaggio regionale ha permesso di arginare meglio che nel resto del Paese il dilagare delle infezioni.

Peculiare risulta infine il caso del Veneto, dove l'evoluzione della malattia è spiegata quasi unicamente dalla componente NE, suggerendo un totale allineamento tra la dinamica della regione e quella media del Paese.

Tra le regioni che occupano una posizione centrale nel Paese (Fig. 2), le Marche si distinguono per un bilanciamento di effetti molto simile a quello del Veneto. In tutte le altre regioni la componente locale (LE) assume un valore positivo non residuale, evidenziando una tendenza dei fattori locali di queste regioni sfavorevole alla diffusione del contagio, che controbilancia in buona misura il portato negativo della componente nazionale (NE).

Tra le regioni meridionali (Fig. 3) l'effetto compensativo tra la componente nazionale NE negativa e la componente locale LE positiva è ancora più marcato. A differenza che nelle altre macroregioni analizzate, il fatto che in termini assoluti i valori di queste componenti quasi si equivalgono fa sì che la curva risultante, rappresentativa della dinamica delle infezioni, rimanga molto bassa. In Basilicata, Sicilia e Calabria il bilanciamento è quasi perfetto e il numero degli infetti risulta, infatti, una quota residuale della popolazione complessiva; ma anche in regioni molto popolate e ricche di attività come la Cam- 

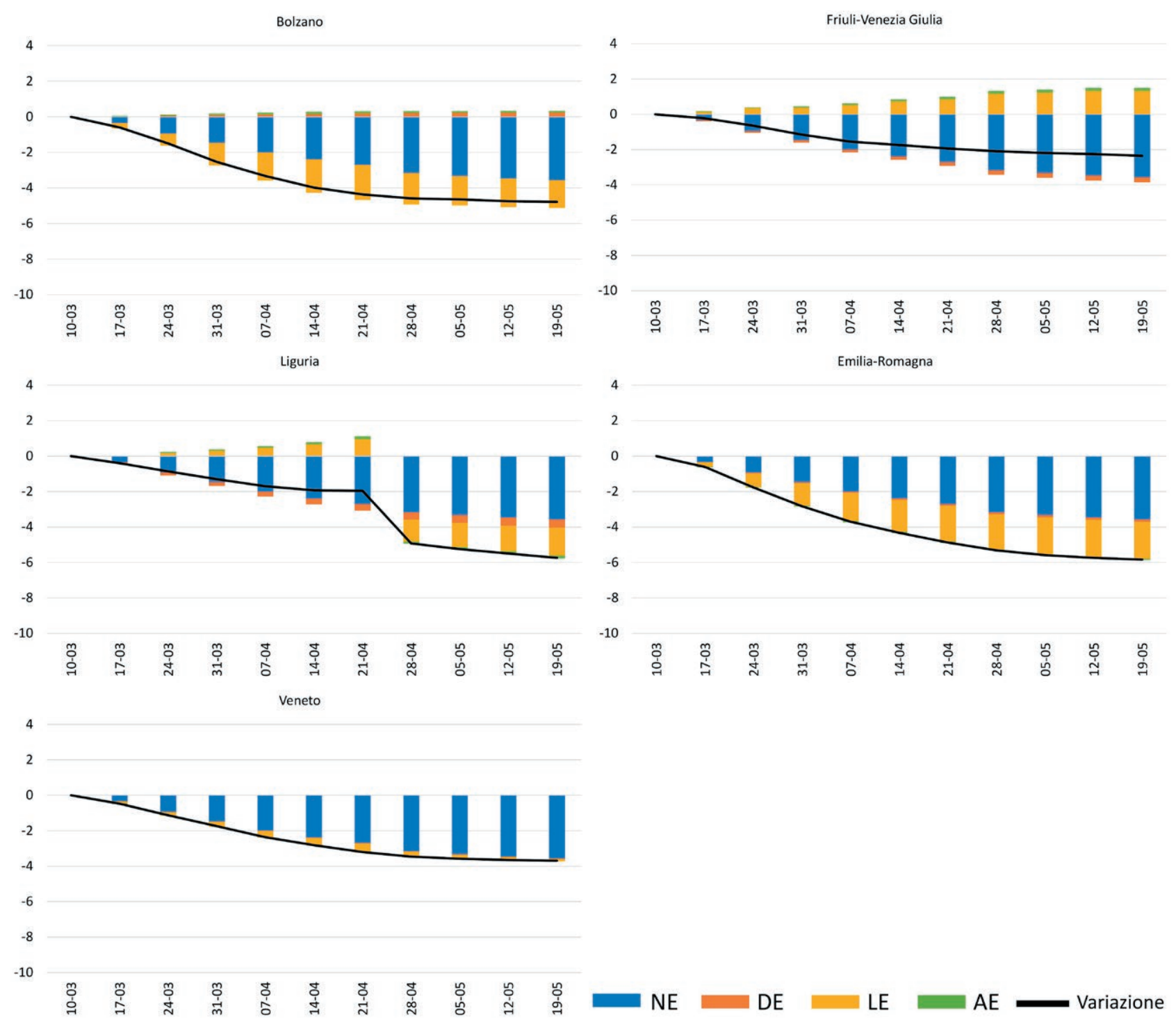

Figura 1b. Shift-share della dinamica delle infezioni da COVID-19 certificate in Italia nelle regioni settentrionali: Valle d'Aosta, Piemonte, Liguria, Lombardia, Provincia Autonoma di Trento, Provincia Autonoma di Bolzano, Veneto, Friuli-Venezia Giulia, Emilia-Romagna. La linea nera rappresenta la diminuzione percentuale del numero di persone non infette. Periodo: 9 marzo - 20 maggio 2020. Fonte: nostre elaborazioni su dati ISS.

pania (e in misura minore la Puglia) la differenza tra le due componenti risulta molto contenuta.

\section{Riflessioni conclusive}

Il lavoro presenta una proposta di adattamento della tecnica di analisi shift-share da applicare allo studio della prima ondata di diffusione delle infezioni da COVID-19. Alla base di questo esercizio pilota vi è la convinzione che la tecnica shift-share, solo di recente introdotta in modo stabile nelle analisi di ambito geografico, offra delle grandi potenzialità di analisi regionale, non solo se applicata allo studio dei differenziali di crescita (così come accade normalmente) ma anche con riferimento ai trend di crescita di variabili non economiche.

Con la riformulazione qui proposta diventa possibile analizzare la diffusione regionale della malattia separando il ruolo giocato dalla struttura demografica regionale (che indica se ed in che misura la variazione nel numero degli infetti sia imputabile alla composizione per fasce d'età), da quello esercitato, in termini aggregati, dall'in- 

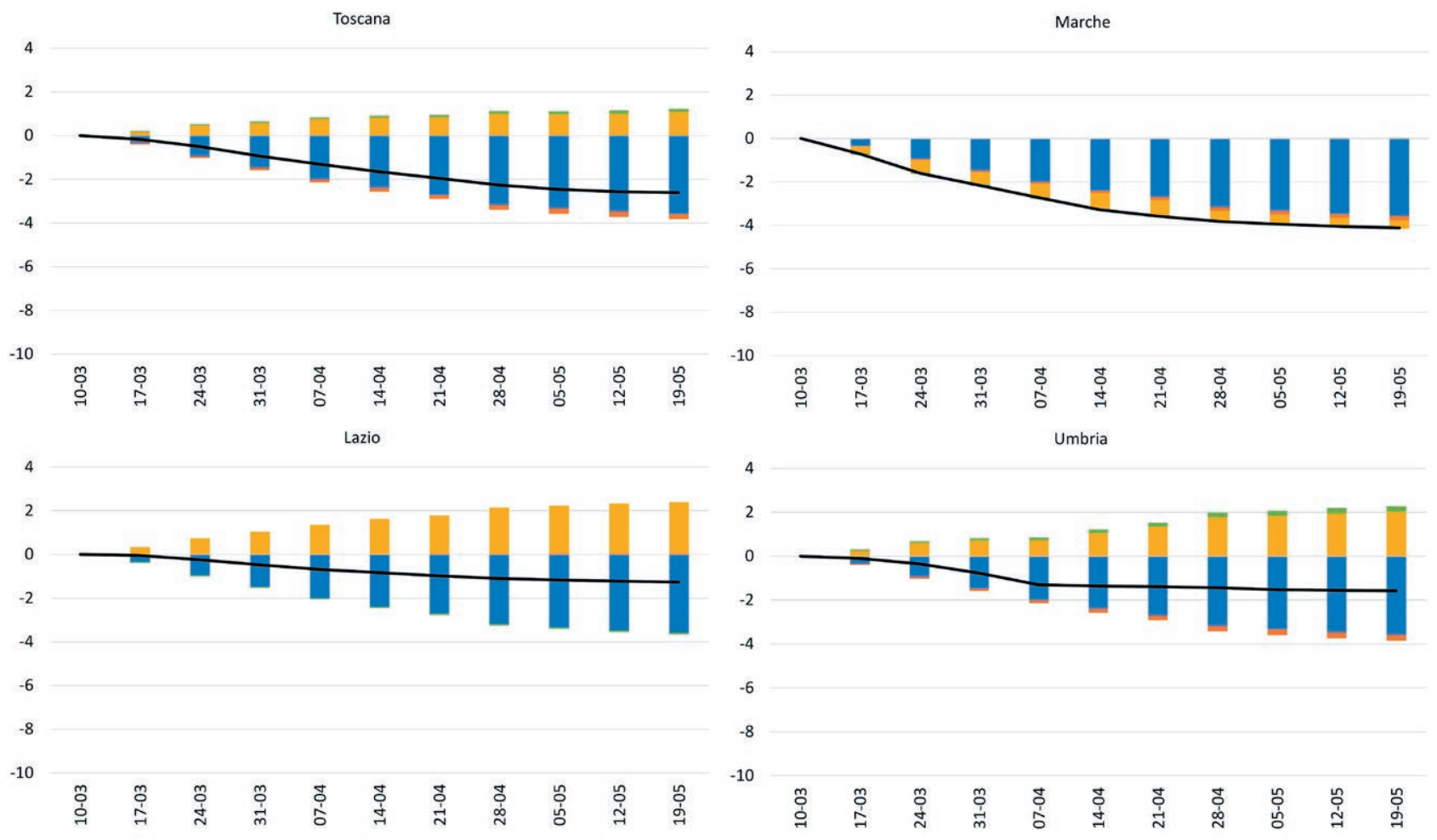

NE DE $\square$ LE

$\mathrm{LE} \quad \mathrm{AE}$

Variazione

Figura 2. Shift-share della dinamica delle infezioni da COVID-19 certificate in Italia nelle regioni centrali: Toscana, Umbria, Marche, Lazio. La linea nera rappresenta la diminuzione percentuale del numero di persone non infette. Periodo: 9 marzo - 20 maggio 2020. Fonte: nostre elaborazioni su dati ISS.

sieme delle altre caratteristiche e fattori endogeni alle regioni (specifiche condizioni territoriali di diffusione del contagio, densità della popolazione, comportamenti individuali e collettivi, politiche di contenimento, ecc.).

Nel proporre questo adattamento - consapevoli della complessità del compito - si è scelto di partire considerando la formulazione classica della SSA che, a differenza delle versioni con pesatura geografica, non tiene conto delle relazioni che si instaurano tra le unità statistiche analizzate, in questo caso le regioni. Se, da un lato, questa scelta implica una semplificazione, dall'altro lato permette di calibrare meglio la trattazione rispetto all'obiettivo assunto nell'articolo. Avendo come riferimento la letteratura geografica e regionalista, lo studio qui condotto non intende fornire risposte definitive, ma suggerire la strada per un nuovo filone di analisi che, proprio per la sua novità, richiede passaggi intermedi e affinamenti successivi.

La riformulazione della shift-share qui proposta ha permesso di separare la dinamica dei contagi in quat- tro componenti: quelle numericamente più consistenti, e quindi maggiormente rappresentative del trend finale, sono quelle che fotografano le condizioni di diffusione della malattia a livello nazionale (NE) e regionale (LE). Le restanti due componenti (AE e DE), che tengono conto della distribuzione della popolazione per le varie coorti di età, risultano nulle o residuali. In una condizione in cui le distribuzioni siano molto diverse tra le regioni, questo risultato porterebbe quindi a negare l'idea, molto diffusa nelle prime settimane della pandemia, che una maggiore presenza di popolazione anziana possa spiegare non solo la maggiore mortalità e letalità dimostrata dal virus in alcune regioni, ma anche le differenze stesse nella diffusione delle infezioni.

Va però chiarito che, ad essere messa in dubbio dalla nostra analisi non è la maggiore incidenza della malattia sugli strati più anziani della popolazione. Quello che i risultati mostrano è che, per le regioni italiane, all'interno del sistema dei condizionamenti che possono influire sulla diversificazione geografica della dinamica 

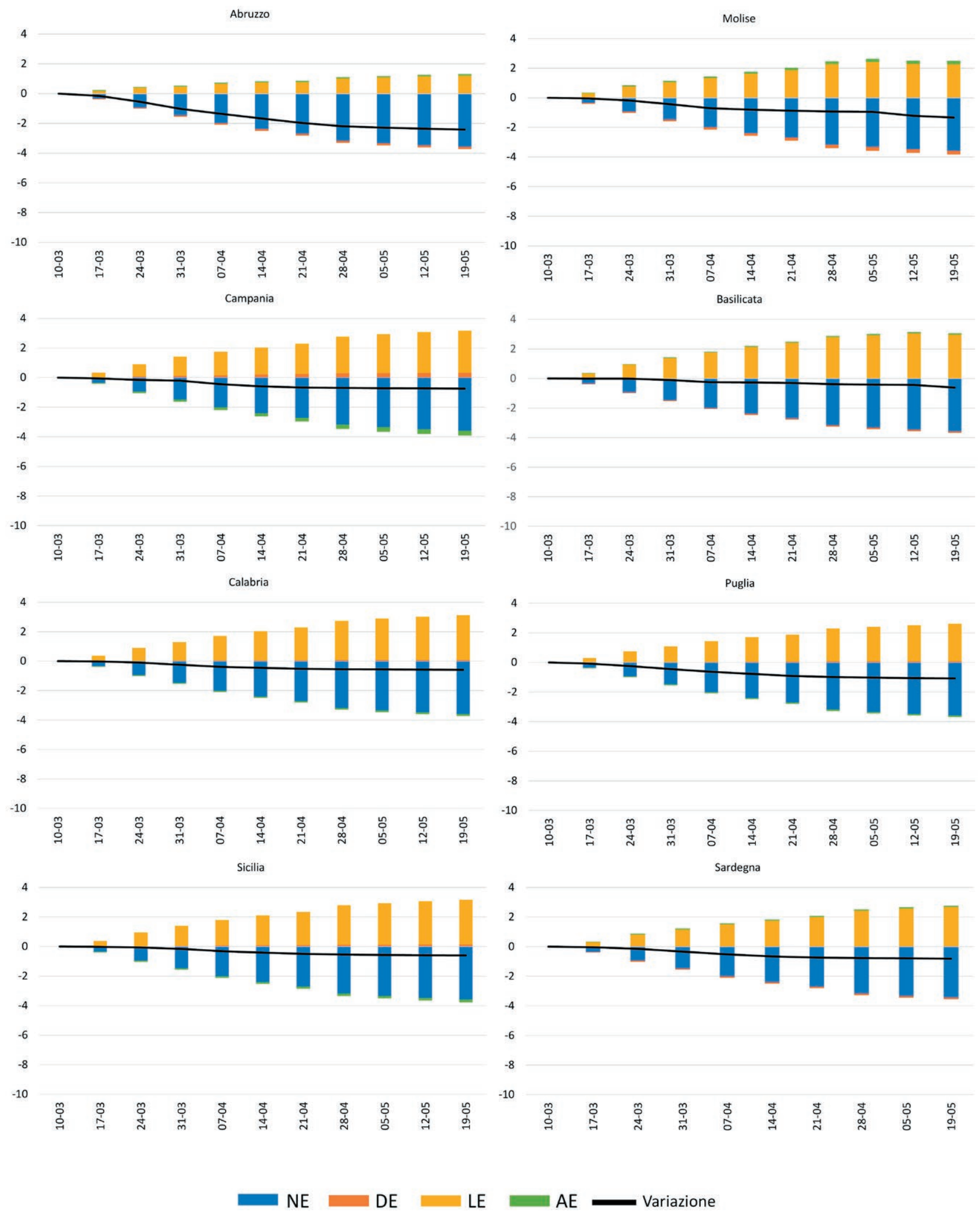

Figura 3. Shift-share della dinamica delle infezioni da COVID-19 certificate in Italia nelle regioni meridionali: Abruzzo, Molise, Campania, Puglia, Basilicata, Calabria, Sicilia, Sardegna. La linea nera rappresenta la diminuzione percentuale del numero di persone non infette. Periodo: 9 marzo - 20 maggio 2020. Fonte: nostre elaborazioni su dati ISS. 


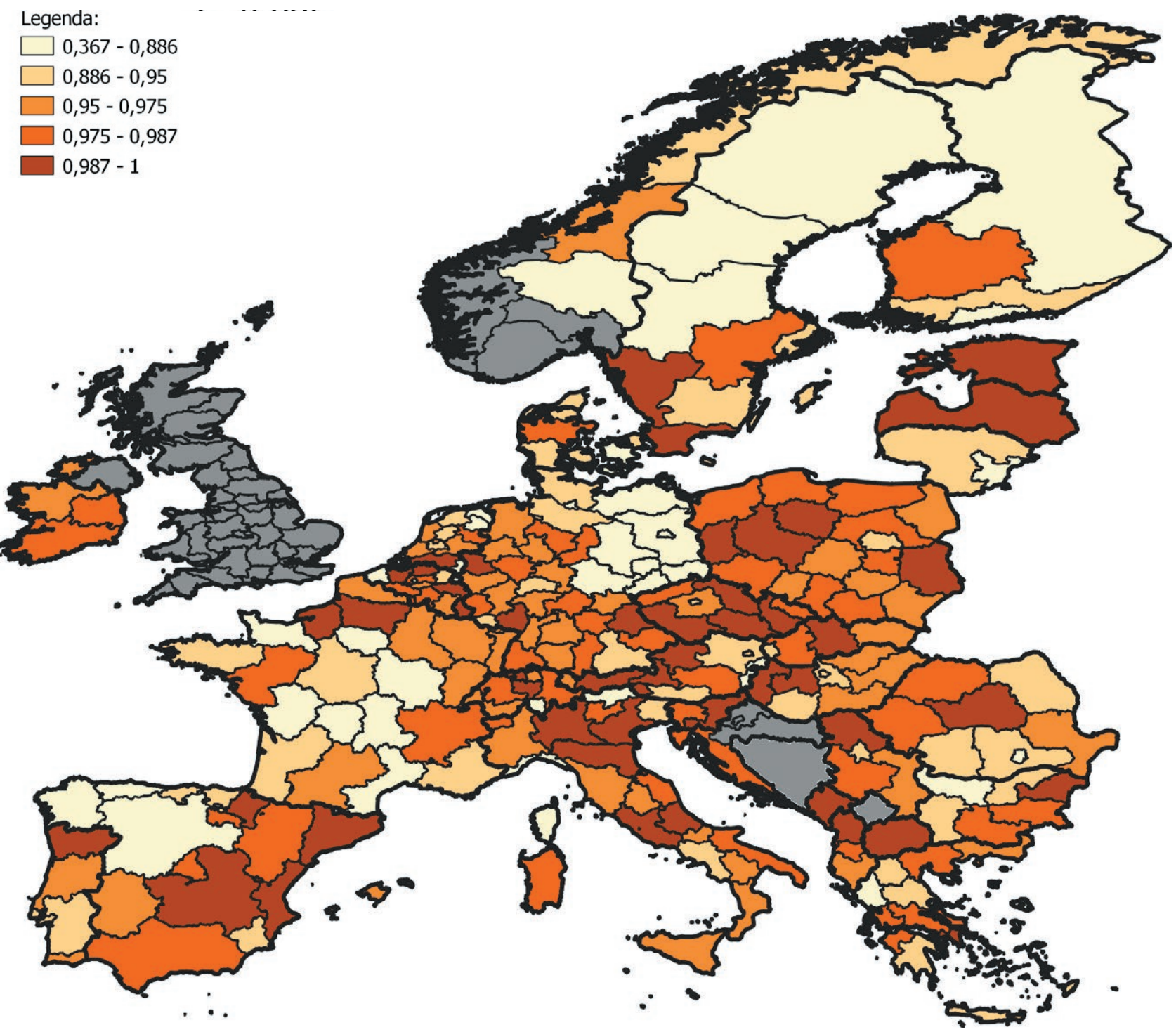

Figura 4. Variabilità della struttura demografica delle regioni europee rispetto alla nazione di appartenenza. L'indice è costruito come il coefficiente di correlazione di Pearson tra la distribuzione per classi di età della popolazione regionale e il corrispettivo valore nazionale. Ripartizione in classi per quintili. Fonte: elaborazione propria su dati Eurostat.

diffusiva del virus SARS-CoV-2, quelli di tipo strutturale derivanti dalla distribuzione per età della popolazione sono residuali rispetto a quelli riferibili alle caratteristiche endogene delle regioni. D’altro canto, la natura descrittiva della metodologia, non permette di sapere se questo accade per un effettivo ruolo limitato della struttura demografica o, più semplicemente, per l'elevata omogeneità della distribuzione della popolazione per fasce d'età delle regioni italiane. Rispetto quindi alle posizioni di quanti riflettono sull'opportunità di misure anti-COVID ritagliate su specifiche classi di età (per esempio: Bignami-Van Assche 2020), allo stato dell'arte della nostra analisi non è possibile assumere altra posizione che non sia quella interlocutoria. Per verificare che i risultati ottenuti non riflettano l'estrema similarità delle strutture demografiche regionali, andrebbero messi a confronto con quelli relativi ad altri paesi e altri contesti demografici. Come si è già avuto modo di sottolineare, una potenziale debolezza metodologica può infatti essere identificata nella scelta del caso di studio, che sfrutta l'opportunità di una serie completa di dati stratificata per classi di età, ma che si è rivelata essere molto omogenea nella distribuzione anagrafica per fasce di età tra le regioni. Questo fatto ha sicuramente ridimensionato 
il potere di lettura della shift-share, che basa la sua efficacia proprio sull'esistenza di significativi differenziali regionali nelle variabili analizzate.

A titolo esemplificativo, nella figura 4 si mostra la variabilità della distribuzione della popolazione regionale per fasce di età in Europa, da cui si evince come la situazione italiana di marcata omogeneità non sia generalizzabile ad altre nazioni europee. In particolare spiccano Francia, Spagna e Germania per l'elevata variabilità interna, candidandosi così a essere promettenti nuovi ambiti di sperimentazione della riformulazione dell'analisi shift-share proposta in questo studio.

La sperimentazione su altri contesti potrebbe anche aiutare a controllare per un altro limite che si osserva in generale con le tecniche di scomposizione e che nel nostro caso consiste nell'influenza esercitata dal diverso peso demografico delle unità territoriali considerate, tale per cui le regioni più popolose influiscono di più sulla struttura nazionale.

Per quel che attiene gli altri effetti della shift-share, la sperimentazione da noi condotta suggerisce che, nel tenere basse le curve delle infezioni, il contributo maggiore sia venuto dal livello regionale o endogeno, misurato attraverso l'effetto LE. Ai fini pratici, ci si scontra però con due ulteriori limiti della shift-share classica: il primo riguarda la dimensione deterministica della formula di scomposizione, che non considera l'influenza dei fattori esogeni, né quella che si produce per effetto delle relazioni tra le regioni; la seconda riguarda la difficoltà di definire in cosa consistano, nel concreto, le caratteristiche endogene delle regioni. Una dimensione di analisi che è centrale nella riflessione geografica, ma che la formulazione classica della shift-share, anche nella sua versione dinamico-cumulativa, non consente di cogliere. Di qui l'interesse per un'ulteriore sperimentazione che prenda in considerazione le elaborazioni più "raffinate" della SSA, a partire dal quelle probabilistiche e spaziali.

L'analisi shift-share offre al ricercatore degli indizi di matrice regionale/territoriale da cui partire per formulare nuove ipotesi e linee di ricerca. In questo modo i risultati potrebbero essere utilizzati per pianificare l'azione politica, che, a sua volta, gioca un ruolo fondamentale nel condizionare le curve epidemiche (Anderson et al. 2020). Nello stesso tempo, la shift-share da sola non consente di capire se, a fare la differenza, siano state le politiche messe in atto dalle amministrazioni territoriali (per esempio molto efficienti nel contenimento iniziale della pandemia sono risultate le misure intraprese dalla Regione Veneto; Meneghesso 2020), quanto piuttosto i comportamenti individuali e collettivi, a loro volta influenzati dalle particolari condizioni di confor- mazione e frequentazione dei luoghi di vita e lavoro. Da qui l'indicazione che analisi quantitative di questo tipo siano sempre affiancate da opportuni approfondimenti qualitativi, da realizzare attraverso analisi documentale e interviste. Così operando, si potrà forse ridurre un po' dell'incertezza che ancora riguarda molti aspetti di questa epidemia e che richiede cautela e approfondimento delle eventuali relazioni causa-effetto.

\section{Riferimenti bibliografici}

Anderson, R.M., Heesterbeek, H., Klinkenberg, D., Hollingsworth, T.D. (2020). How will country-based mitigation measures influence the course of the Covid-19 epidemic? Lancet, 395(10228), 931-934. DOI: https://doi. org/10.1016/S0140-6736(20)30567-5

Artige, L., van Neuss, L. (2014). A new shift-share method. Growth Change, 45, 667-683. DOI: https://doi. org/10.1111/grow.12065

Ascani, A., Faggian, A., Montresor, S. (2021). The geography of COVID-19 and the structure of local economies: The case of Italy. Journal of Regional Science, 61(2), 407-441, DOI: https://doi.org/10.1111/jors.12510

Bagliani, M., Feletig, P., Ferlaino, F., Rota, F.S. (2019). Proposta di analisi shift-share dinamico-cumulativa al caso delloccupazione delle metroregioni italiane (20002014). In Lattarulo P. et al. (a cura di). Le Regioni d'Europa tra identità locali, nuove comunità e disparità territoriali. Milano, Franco Angeli, 119-142.

Barff, A., Knight, P. (1988). Dynamic shift-share analysis. Growth Change, 19, 2-10. DOI: https://doi. org/10.1111/j.1468-2257.1988.tb00465.x

Bignami-Van Assche, S., Ghio, D., Van Assche, A. (2020). Not just a concern for the elderly: Age gradient in Covid19-related infections in Italy, Spain and the Netherlands. CIRANO Working Papers, 2020s-17. https://ideas.repec. org/p/cir/cirwor/2020s-17.html

Bristow, G. (2010). Resilient regions: Re-'place'ing regional competitiveness. Cambridge Journal of Regional Economy and Society, 3, 153-167. DOI: https://doi. org/10.1093/cjres/rsp030

Cohen, J., Kupferschmidt, K. (2020). Countries test tactics in 'war' against Covid-19. Science, 367(6484), 12871288, DOI: $10.1126 /$ science.367.6484.1287

Conti Puorger, A. (2018). Analisi geografica e metodi statistici. Una introduzione. Bologna, Pàtron.

Daniel, W.W., Cross, C.L. (2019). Biostatistica. Concetti 
di base per l'analisi statistica delle scienze dell'area medicosanitaria. Napoli, Edises.

Dowd, J.B., Andriano, L., Brazel, D.M., Rotondi, V., Block, P., Ding, X., Liu, Y., Mills, M.C. (2020). Demographic science aids in understanding the spread and fatality rates of Covid-19. PNAS, May 5 2020, 117(18) 9696-9698. DOI: https://doi.org/10.1073/pnas.2004911117

Dudel, C., Riffe, T., Acosta, E., van Raalte, A., Strozza, C., Myrskylä, M. (2020). Monitoring trends and differences in COVID-19 case-fatality rates using decomposition methods: Contributions of age structure and age-specific fatality. PLoS ONE, 15(9), e0238904. DOI: https://doi. org/10.1371/journal.pone.0238904

Dunn, E.S. (1959). Une technique statistique et analytique d'analyse régionale: description et projection. Économie appliquée, 4, 521-530.

Esteban-Marquillas, J.M. (1972). A reinterpretation of shift-share analysis. Regional and Urban Economics, 2, 249-261. DOI: https://doi.org/10.1016/00343331(72)90033-4

Fernàndez, M.M., Menàndez, A.J.L. (2005). The spatial shift-share analysis - new developments and some findings for the Spanish case. ERSA conference papers ersa05p659, https://ideas.repec.org/p/wiw/wiwrsa/ ersa05p659.html

Istituto Nazionale di Statistica (ISTAT) (2018). Spostamenti quotidiani e nuove forme di mobilità. Comunicato stampa. https://www.istat.it/it/archivio/224469

Istituto Nazionale di Statistica (ISTAT) (2020). Aspetti di vita degli over 75. https://www.istat.it/it/files//2020/04/ statisticatoday_anziani.pdf

Istituto Superiore di Sanità (ISS) (2020a). Appendici statistiche del periodico Bollettino sull'epidemia COVID-19, rilasciato dall'Istituto Superiore di Sanità dal 9/03/2020 al 20/05/2020. https://www.epicentro.iss.it/coronavirus/bollettino

Istituto Superiore di Sanità (ISS) (2020b). Epidemia COVID-19. Aggiornamento nazionale 20 maggio 2020 ore 16:00. https://www.epicentro.iss.it/coronavirus/ bollettino/Bollettino-sorveglianza-integrata-COVID19_20-maggio-2020.pdf

Kluge, H.H.P. (2020). Statement - Older people are at highest risk from Covid-19, but all must act to prevent community spread. World Health Organisation (WHO). https://www.euro.who.int/

Kubota, Y., Shiono, T., Kusumoto, B., Fujinuma, J. (2020). Multiple drivers of the COVID-19 spread: The roles of climate, international mobility, and region-specific conditions. PLoS ONE, 15(9), e0239385. DOI: https://doi. org/10.1371/journal. pone.0239385

Lahr, M.L., Ferreira, J.P. (2020). A reconnaissance through the history of shift- share analysis. In Batey P. (eds.). Handbook of Regional Science. New York City, Springer. DOI: http://dx.doi.org/doi:10.7282/t3-0gs3-nw29

Lloyd-Sherlock, P., Sempe, L., McKee, M., Guntupalli, A. (2021). Problems of data availability and quality for COVID-19 and older people in low- and middle-income countries. The Gerontologist, 61(2), 141-144. DOI: https://doi.org/10.1093/geront/gnaa153

Lombardo, F.L., Bacigalupo, I., Salvi, E., Lacorte, E., Piscopo, P. Mayer F., Ancidoni, A., Remoli, G., Bellomo, G., Losito, G., D’Ancona, F., Bella A., Pezzotti, P., Canevelli, M., Onder, G., Vanacore, N. (2020). The Italian national survey on Coronavirus disease 2019 epidemic spread in nursing homes. International Journal of Geriatric Psychiatry. DOI: https://doi.org/10.1002/ gps.5487

Meneghesso, G., Garzotto, F., Rizzuto, R., Vettor, R. (2020). Covid-19: Effectiveness of widespread diagnostic tests to prevent health care collapse in the Veneto Region, Italy. The Lancet Public Health - SSRN. DOI: http://dx.doi. org/10.2139/ssrn.3576942

Musolino, D., Rizzi, P. (2020). Covid-19 e territorio: un'analisi a scala provinciale. EyesReg, 10(3). http://www. eyesreg.it/2020/covid-19-e-territorio-unanalisi-a-scalaprovinciale/

Nazara, S., Hewings, G.J.D. (2004). Spatial structure and taxonomy of decomposition in shift-share analysis. Growth and Change, 35(4), 476-490. DOI: 10.1111/j.1468-2257.2004.00258.x

Nepomuceno, M.R., Acosta, E., Alburez-Gutierrez, D., Aburto, J.M., Gagnon, A., Turra, C.M. (2020). Besides population age structure, health and other demographic factors can contribute to understanding the Covid-19 burden. PNAS, 117(25), 13881-13883. DOI: https://doi. org/10.1073/pnas.2008760117

Patterson, M.G. (1991). A note on the formulation of a full-analogue regression model of the shift-share method. Journal of Regional Science. 31(2), 211-216. DOI: https:// doi.org/10.1111/j.1467-9787.1991.tb00143.x

Pisano, G.P., Sadun, R., Zanini, M. (2020). Lessons from Italy's Response to Coronavirus. Harvard Business Review, March 27 2020. https://hbr.org/2020/03/lessons-fromitalys-response-to-coronavirus 
Rota, F.S., Bagliani, M., Feletig, P. (2020). Breaking the black-box of regional resilience: A taxonomy using a dynamic cumulative shift-share occupational approach. Sustainability, 12(21), 9070. DOI:_https://doi.org/10.3390/ su12219070

Sarti, R. (2010). Who cares for me? Grandparents, nannies and babysitters caring for children in contemporary Italy. Paedagogica Historica, 46(6), 789-802. DOI: https:// doi.org/10.1080/00309230.2010.526347

Sihag, B.S., McDonough, C.C. (1989). Shift-share analysis: The international dimension. Growth Change, 20, 80-88. DOI: https://doi.org/10.1111/j.1468-2257.1989. tb00497.x

Wolkewitz, M., Puljak, L. (2020). Methodological challenges of analysing COVID-19 data during the pandemic. BMC Medical Research Methodology, 20(1), 81. DOI:10.1186/s12874-020-00972-6 